\title{
Recent progress in the control of fish behaviour:
}

\section{J. G. BALCHEN $\dagger$}

Keywords: fish behaviour, control, conditioning, control stimuli, acoustical repulsion, long term storage, fish farming, electrical barrier.

\begin{abstract}
The paper gives a survey of recent activities in developing techniques for controlling fish behaviour over short and medium distances. The bases for control are described with reference to the sensory system of the fish and a simple model of fish behaviour. It is suggested that an effective way of controlling fish behaviour is by means of conditioning, in which reward or punishment is used in connection with an acoustical or visual signal. A number of possible applications which have been tested in practice are described.
\end{abstract}

\section{Introduction}

Man has been able to gain control over many animals on the earth. This has led to the domestication of many species for the purpose of food production, transportation, herding, etc.

The relationships between fish and man, however, have been rather simple. Man has usually regarded fish as something existing in a dark and alien world mostly unobserved by the human eye and beyond human control. Fishing techniques employed around the world clearly indicate that man sets up his fishing gear so that it interacts with a natural behaviour of the fish, such as feeding and spawning migration, in order to arrive at a high probability of catch. Rarely do men attempt to control the behaviour so as to maximize the amount of fish caught per unit fishing effort.

The increased intensity of exploitation of fish resources in recent years has led to a need for a better understanding of fish behaviour and for methods for controlling fish behaviour so as to arrive at a more useful interaction between the human and the fish. Such an interaction may have to do with harvesting the natural yield of the fish resources, herding the fish during marine farming and, possibly, modification of migration behaviour with the aim of improving the ecological system performance. In order to make progress in the field indicated above, it is believed that the application of some of the powerful results of modern systems theory will prove to be fruitful.

It is the purpose of this paper to describe some aspects of the control of fish behaviour from the point of view of systems theory and to describe some practical applications that have been derived in recent years.

\section{Fish behaviour}

This paper deals with behaviour in terms of movement over short or medium distances, ranging from a few metres to hundreds of metres. Behaviour in terms of 'tail wiggling' is of no concern here.

Received 10 December 1982

$\dagger$ Division of Engineering Cybernetics, The Norwegian Institute of Technology, Trondheim, Norway.

¥ Paper presented at IFAC Eighth World Congress, Kyoto, Japan 24-28 August 1981. 
The behaviour of schooling fish is of greatest interest because the tendencies of the individual in a school are suppressed by the behaviour of the entire school. The result is that the behaviour of the school is more amenable to description and prediction (Balchen, 1972; Radakov, 1973).

The natural behaviour of fish is the outcome of an interaction between the physiological state of the individuals and the state of the environment (Balchen, 1975; Balchen, 1979). The physiological state of an individual fish (or a school of identical individuals) is a set of the least number of time variable quantities which gives a sufficient description of the physiological functions of the fish with regard to behaviour. These dynamic variables (state variables) can be related by a number of coupled first order differential equations equal to the number of state variables. The state of the environment can also be described by a set of dynamic variables which give a sufficiently detailed representation of the environment with respect to fish behaviour. Typical variables belonging to the state of the environment are temperature, food concentration, intensity of light, intensity of predators, water currents, etc. (Balchen, 1976a).

The interaction between the physiological state of the fish and the state of the environment which determines the behaviour of the fish is suggested to be of the following form (Balchen, $1976 \mathrm{~b}$ ):

The physiological state of the fish $(x)$ and the state of the environment $(v)$ together produce a scalar field of stimuli $(C(x, v))$ which can be defined as 'comfort' or 'discomfort'. The fish will at any time move $(\dot{r})$ so as to maximize this 'comfort'.

This interpretation of fish behaviour fits very well with the observed behaviour in the ocean: fish are attracted by olfactory stimuli (scalar field of chemical agent concentration); fish are repelled by both low and high temperatures and find maximum comfort at a certain intermediate temperature; fish at a certain stage and physiological state are repelled by light and go deep; and fish are either repelled (frightened) by certain sounds which can be identified with danger or perhaps attracted by other sounds which they have learned to identify with something desirable.

A simple mathematical model which describes the active movement of the fish is

$$
\dot{\boldsymbol{r}}=\frac{\partial C(\boldsymbol{x}, \boldsymbol{v}(\boldsymbol{r}))}{\partial \boldsymbol{r}}
$$

in which $\boldsymbol{r}$ is fish location vector.

Active control of fish behaviour can, in principle, be achieved in a number of different ways:

Generating artificial stimuli which either attract or repel the fish.

Modifying the physiological state of the fish so that the natural stimuli appear differently.

Modifying the environment, thereby producing modified stimuli.

\section{Basis for control of fish behaviour}

The effectiveness of control-the degree of obeying an externally applied behaviour reference rather than the natural behaviour-is highly dependent upon the sensitivity (gain) which can be achieved for the control stimuli relative to the natural stimuli. The sensitivity to natural stimuli changes drastically with time due to changes in the 
physiological state of the fish. An example of this is the sensitivity to food stimuli which simply is dependent upon the degree of satiation. This in turn depends upon the intensity and timing of previous feeding. The attraction of fish to food stimuli will obviously be influenced by such factors.

One important aspect of behavioural control is the utilization of the tendency among most species to imitate the behaviour of other individuals. Imitation is one of the basic phenomena behind schooling of fishes. Observers of fish behaviour know that if one or a few fish are seen by other fish rushing towards something, the others will immediately do the same. They in turn cause other fish further away to do likewise. Consequently the transmission of information may far exceed the limited range of direct visual contact (Balchen, 1972; Manteyfel, 1978; Radakov, 1973). One way to increase the sensitivity to external control stimuli will thus be to arrange for the possibility of imitation, thereby producing a self-generated increase in gain.

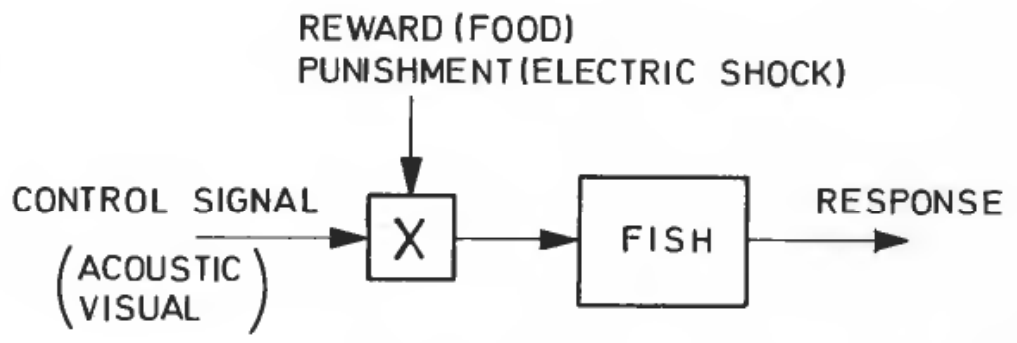

Figure 1. Simplified model of 'conditioning' for fish behaviour control.

Probably the most important way of achieving that a fish obeys a control signal is to use conditioning and the ability of the fish to learn (Bull, 1957). The practical realization of conditioning may be in the form of a combination of two or more types of signals, one which is easy to generate and which propagates fast and far in the water masses (sound signal, visual signal) and another which in fact may take the form of a reward or a punishment (food, electrical shock). The application of either one of the two types of signals separately may give a very weak response whereas the combination is very effective. In Figure 1, it is indicated in a block diagram how the combination of two types of stimuli may be 'modeled' as a multiplication or a 'logical and'. This type of control method will be dealt with in detail in a later section. Commonly in the control of vehicles, industrial processes, etc. feedback from measurements indicating the systems performance plays an important role. In controlling fish behaviour it would also be advantageous to establish feedback to modify the control stimuli according to the deviation between the desired behaviour and the observed behaviour. But measurement of fish behaviour may prove to be rather difficult. In case a direct measurement cannot be made, one is forced to produce the control stimuli according to predicted or estimated behaviour. The resulting control system will be a combination of feedback and feedforward control, since it combines modeled (predicted) behaviour with measured behaviour.

\section{Control stimuli}

Due to space limitations we shall only give a listing of the most important stimuli with some comments as follows: 
With regard to direction: (Balchen, 1976c).

Attracting stimuli (smell of food, certain sounds) and repelling stimuli (certain sounds, certain smells).

Directional (vectoral) stimuli (sound, light) and nondirectional (scalar) stimuli (temperature, smell of food).

Converging stimuli (point source of attractive stimuli) or diverging stimuli (point source of repulsive stimuli).

With regard to physical origin:

Acoustic stimuli:

Mechanical vibrations in the water in the frequency range $10-500 \mathrm{~Hz}$ which can be detected by a number of different sensing organs i.e. the lateral line, the three otholites, etc. (Dijkgraaf, 1962).

'Biological sounds' (e.g. feeding sound), having a particular meaning to the fish, giving either attractive or repulsive stimuli (Maniwa, 1966).

Acoustical signal in connection with conditioning system (Balchen, 1976 c).

Visual stimuli: (Protasov, 1970)

General light intensity.

Particular light source producing a directional signal in connection with conditioning.

Geometrical figures yielding either attractive or repulsive stimuli.

Olfactory stimuli: (Døving et al., 1973)

Source of biochemical agent giving either attractive or repulsive stimulus (mostly nondirectional) (e.g. pheromones).

Electrical stimuli:

Weak electrical sources which can be detected by specialized organs in some species (Fessard, 1974).

Strong electrical sources giving electrical shocks yielding electrotaxis (Sternin et al. 1976) or 'punishment' in systems for conditioning (Balchen, $1976 \mathrm{c}$ ).

Many more types of stimuli are certainly active in the natural life of fish and must be taken into account when considering control. Some of those mentioned above are utilized in the practical applications described in the following paragraphs.

\section{Systems for control of fish behaviour}

The methods for generating control stimuli listed above may be incorporated into a number of different kinds of systems for different purposes. Below, some practical control systems will be discussed in some detail.

\section{Control of schooling species (e.g. herring) by acoustical repulsion}

Many schooling species react to low frequency sound. A system for pelagic control of a school of fish may be designed as shown in the block diagram in Figure 2. 


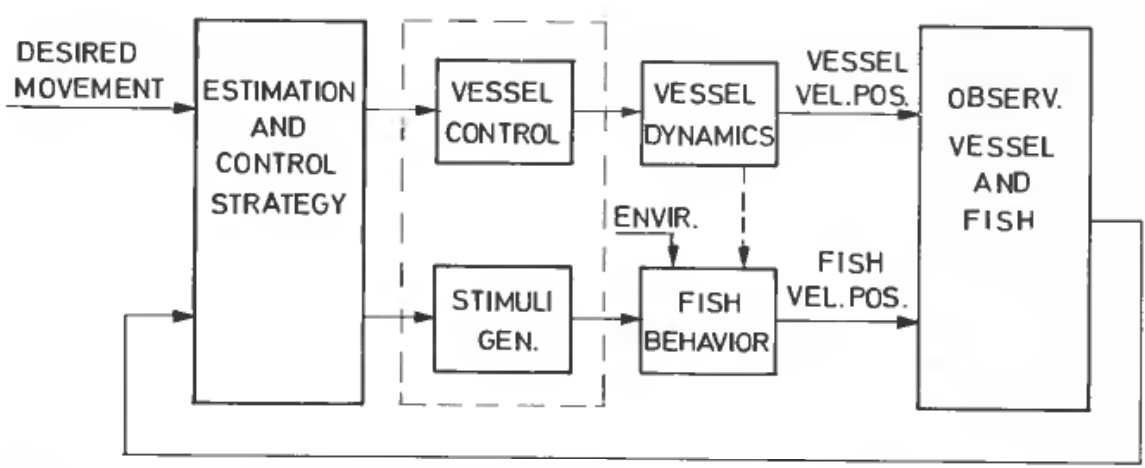

Figure 2. Block diagram of a system for control of schooling species by acoustical repulsion.

The principle employed in this scheme has been partly tested in an experimental set-up (Dalen, 1973). In this diagram, a low frequency acoustic generator is installed on board a vessel which can be manoeuvred to any desired position relative to the fish. It is assumed that the vessel is equipped with a means for measuring the vessel velocity and position as well as the position of the school of fish (depth and horizontal coordinates relative to the vessel). The school of fish in its uncontrolled mode of behaviour will be subject to a number of different stimuli (gradients of food, temperature, light, etc.), the net effect of which is represented by a stimulus vector referred to in Figure 3 as $\boldsymbol{v}_{N}(N=$ natural). According to the theory of fish behaviour (Balchen, $1976 \mathrm{c}$ ) the school will swim in the direction of this natural stimulus vector with a velocity proportional to the magnitude of the vector. When an artificial stimulus (e.g. low frequency sound) is applied, having a directional effect represented by the vector $v_{S}\left(S=\right.$ stimulus), the net effect of the two stimuli $v_{N}$ and $v_{S}$ is computed geometrically as shown in Figure 3. The net direction and velocity of movement will thus be given by the vector $\boldsymbol{v}_{M}(M=$ movement $)$. If the desired direction and velocity of movement $v_{D}\left(D=\right.$ desired) coincide with $v_{M}$, then the stimulus $v_{S}$ has been properly chosen.

The geometrical summation of the two vectors, as shown in Figure 3, is certainly an oversimplification because the occurrence of the artificial stimulus will modify the

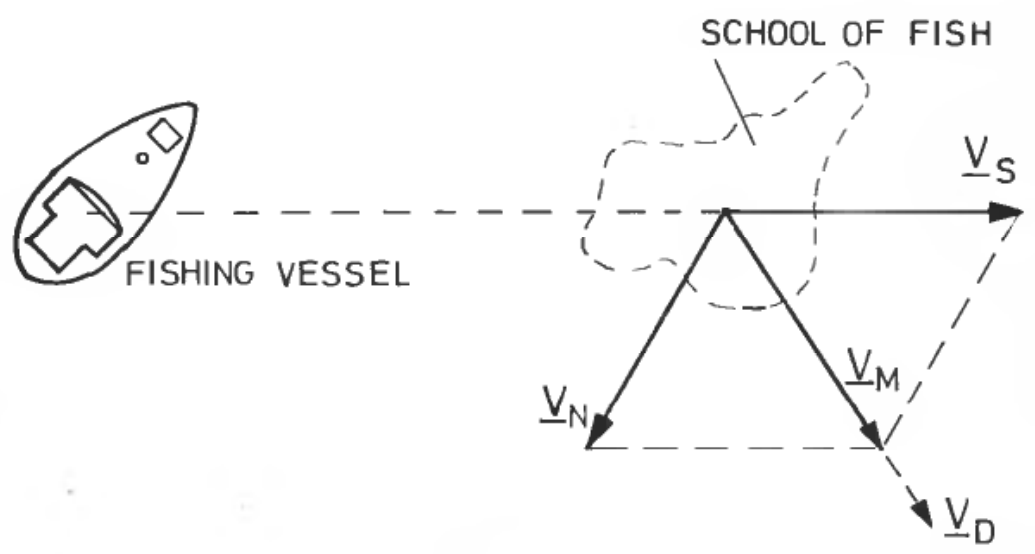

Figure 3. Determination of fish movement by vector summation. 
significance of the natural stimuli. The concept in Figure 3, however, is useful as guide in determining the strength and direction of the artificial stimulus.

Since the stimulus source is located on board the vessel it must be moved to the proper position as indicated in Figure 3. This presents a problem when the magnitude of $v_{N}$ (strong, natural stimuli) and the distance from the vessel to the school are large because the vessel must make large movements to change the direction of $v_{\boldsymbol{s}}$.

This example of control of fish behaviour by repulsion illustrates that it would be a lot simpler if an attractive stimulus were available, which would make the fish converge to the source of the signal rather than diverge away from the source.

The block diagram of Figure 2 is not necessarily only one of a technical system, but could illustrate the functions performed in a system controlled by man. If the control strategies are developed and executed by the human mind, the concept of state of the vessel and of the fish is of major significance. A state estimator, as indicated in Figure 2, which utilizes the measurements and models of vessel dynamics and fish dynamics, is essential if a human control system is to be successful.

Control of fish behaviour using conditioning with sound light and food and applied to long terms storage and farming in large areas

In Norway two particular applications of control of fish behaviour have been under intensive study during the last 5-8 years:

Harvesting of fish (pollock, cod etc.) which have been placed in large, fenced-off bays for long term storage (September-March) to facilitate a constant supply of raw materials to the freezing industry. Conditioning with sound/light and food yields a possibility for 'the fish swimming voluntarily into the factory'. (Balchen, 1978).

Farming of salmon and other expensive species in salt water is most commonly done in rather narrow enclosures (pens). Conditioning by means of sound/light and food yields a possibility for letting the fish move in a larger, fenced-off area $\left(10^{5}-10^{6} \mathrm{~m}^{2}\right)$ and still keeping the fish under control for feeding, inspection, medication and harvesting. (Balchen, 1977; Mork and Balchen, 1980).

\section{Long term storage}

Figure 4 shows a set-up used for conditioning saith or pollock (Gadus virens), which is brought into a fenced-off bay by fishermen for long term storage. Reharvesting the fish (200-1000 tons) by means of conventional fishing gear has proven not to be simple because the fish will be scared and avoid the gear. The system consists of either a number of feeding units floating on the surface or one feeding unit which can be hauled across the bay. Underneath the feeding unit is a loudspeaker and a light source. An electronic control unit produces a programmable sequence of sound pulses in the loudspeaker and light flashes (intensity relative to ambient light), followed by one or more small portions of food from a container. If this sequence is repeated identically with a time interval of, say, $0 \cdot 5-1$ hour, the fish will become conditioned with the result that they behave with great confidence and come into a box shaped net $\left(50 \times 50 \mathrm{~m}^{2}\right)$. The volume of this net can be reduced by mechanical means so that the fish are finally concentrated in a very small volume from which they are directly lifted into the processing plant as shown in Figure 4. The time needed for conditioning depends on many factors, but experiments indicated that under good conditions only a few hours are necessary to obtain good control. It is very important that the fish 


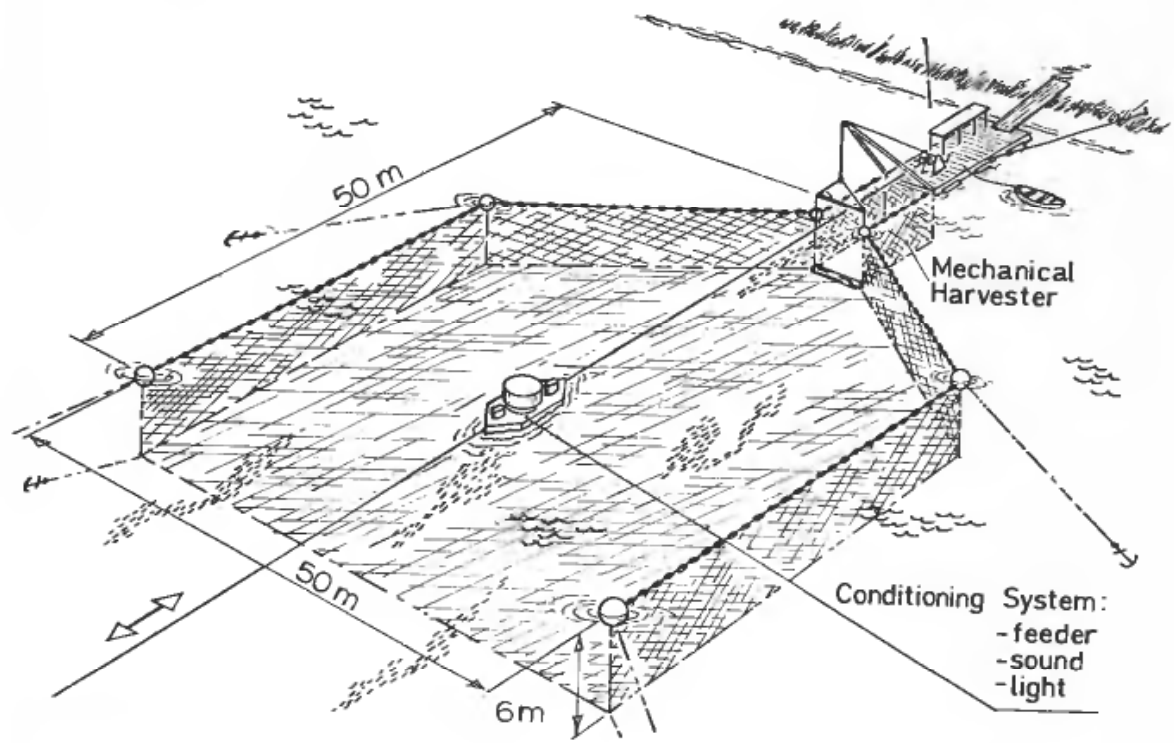

Figure 4. System for reharvesting fish by conditioning during long term storage.

always experience the same type of signals, in other words, it takes time to adapt to a new signal such as a person appearing in a boat in the bay.

The above type of system is very well suited for complete automation by fairly simple technical devices. This is essential since manual reharvesting of the fish by conventional means would cost more than the original harvesting and thus make long term storage uneconomical.

\section{Fish farming}

Principles similar to those described above have been tested for use in connection with large scale farming of salmon in fenced-off bays. The basic idea behind this concept is that one would like to avoid artificial barriers (nets, pens), in which the fish have a high concentration, but rather utilize natural enclosures like bays and sounds. Also it is important that natural exchange of water by tidal movement can be utilized only when the density of fish is fairly small and the means for fencing off has very low resistance to water movement (see description of electrical barriers below). When the fish are spread over a fairly large area $\left(10^{5}-10^{6} \mathrm{~m}^{2}\right)$, there is a need for control of their behaviour in order to monitor the fish quantity, quality, growth, diseases and parasites. Further, there is a need for separating and removing other species competing for the food and, finally, when time has come for slaughter, for collecting the fish in an efficient manner. For this purpose, conditioning by means of sound/light and food has been shown to be very effective (Mork and Balchen 1980).

\section{Fencing off a bay using conditioning with electrical shocks and light pulses}

One of the technical problems of long term storage of fish in a bay and of farming fish in open waters is to prevent the fish escaping. The simplest approach using ordinary nets has certain serious limitations connected with the load of organic material growing on the net and collection of drifting matter. Also if a hole appears in such a net, large amounts of fish will escape. 
In order to produce a barrier which would allow for free exchange of water due to tidal forces and possibly free passage of boats, a system utilizing conditioning with light pulses and electrical shock has been particularly developed in Norway (Balchen, 1976 c; Sølvberg, 1977). A pulsed electrical field set up between electrodes in the water will result in the sensation of pain which will make the fish respond directionally if they have a clue to where the source of the pain is located. Without such a clue, the fish will need longer time to discover the geometry of the system of electrodes producing the electrical potential.

The system which has been tested for both pollock and salmon is shown in Figure 5. Alternating current $(50 \mathrm{~Hz})$ is fed through a heavy cable to electronic control boxes containing thyristors which connect heavy iron electrodes extending vertically from the surface to the bottom. Near the electrodes are fluorescent tubes which emit light pulses at the same instances as the electric current pulses are discharged between the adjacent electrodes.

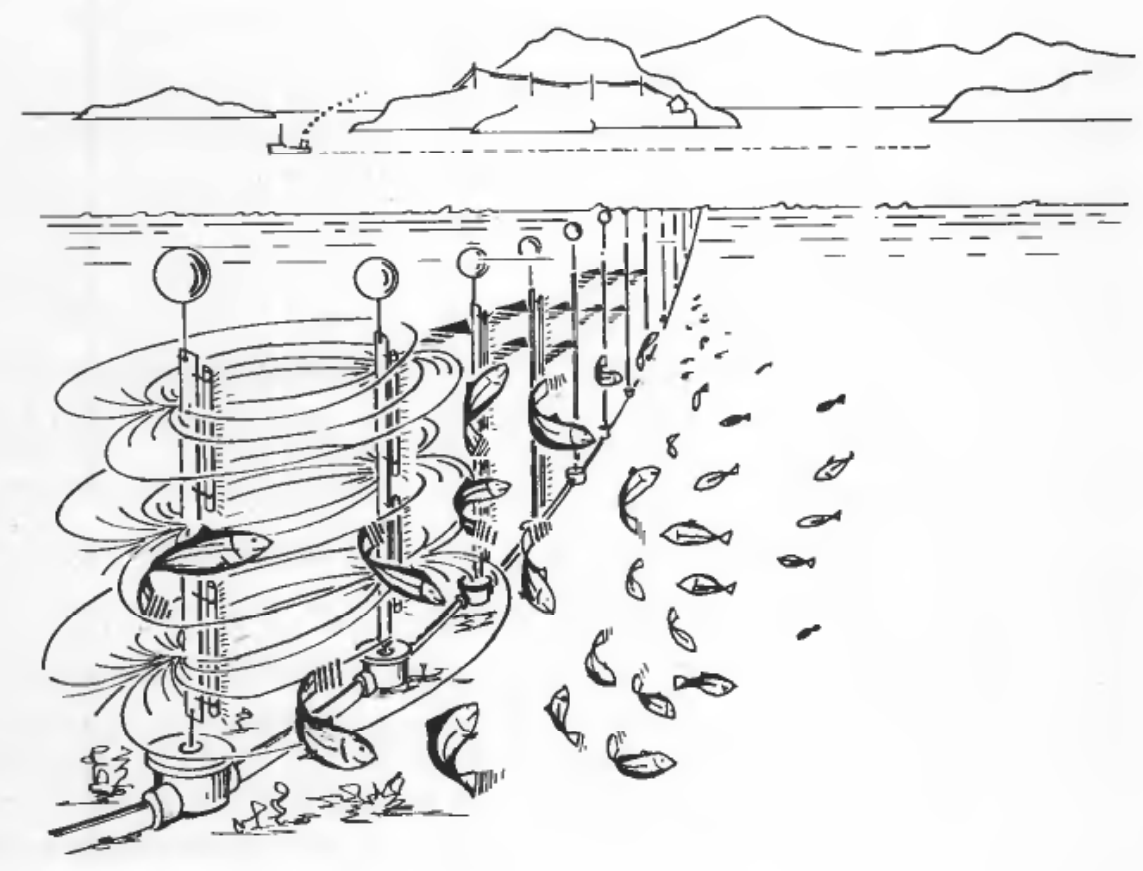

Figure 5. Electrical barrier using conditioning with light.

The electronic switching circuitry is so arranged that part of one half cycle of the alternating current $(50 \mathrm{~Hz})$ is discharged between two neighbouring electrodes, part of the next half cycle between the next two electrodes, and so on. The repetition rate is $1 \mathrm{pulse} / \mathrm{sec}$.

The electrical potential necessary in the water for the fish to experience an uncomfortable shock is dependent upon species, size and orientation of the fish with respect to the electrical field, etc. Many commercial species show a pronounced reaction when they are subjected to an electrical field yielding a 'body potential' of $0.5-2.0$ volts. Setting up electrical fields of this strength is no problem in fresh water, but leads to 
fairly large power consumption in salt water due to the high conductivity of salt water. However, the power consumption is still acceptable from an economic point of view.

It is expected that this type of electrical barrier will have important applications in future aquaculture systems.

\section{Conclusion}

A review has been given of some applications of techniques for controlling fish behaviour. Many more applications can be suggested and it is expected that further development will take place in the near future.

\section{REFERENCES}

Balchen, J. G. (1972). Feedback Control of Schooling Fish. Proc. IFAC 5th World Congr. Pt. I. Instr. Soc. of Am., Pittsburgh, Pa.

Balchen, J. G. (1975). Mathematical Modelling of Fish Behaviour. Principles and Applications. Proc. IFAC 6th World Congr. (Pittsburgh, Instr. Soc. of Am.).

BALCHEN, J. G. (1976 a). Modelling of the Biological State of Fishes. STF48 F76023. Found. of Scient. and Ind. Research (SINTEF), Trondheim, Norway.

Balchen, J. G. (1976 b). Principles of Migration in Fishes. STF48 A76045. Found. of Scient. and Ind. Research (SINTEF), Trondheim, Norway.

Balchen, J. G. (1976 c). Control of Fish Behaviour. STF48 A76082. Found. of Scient. and Ind. Research (SINTEF), Trondheim, Norway.

BALCHEN, J. G. (1977). Farming of Salmon in Large Fenced off Areas. STF48 F77007. Found. of Scient. and Ind. Research (SINTEF), Trondheim, Norway (in Norwegian).

BALCHEN, J. G. (1978). Methods for Reharvesting of Fish Under Long Term Storage. STF48 F78060. Found. of Scient. and Ind. Research (SINTEF), Trondheim, Norway (in Norwegian).

Balchen, J. G. (1979). Modeling, Prediction and Control of Fish Behaviour. p. 99-146, in Control of Dynamic Systems, Ed. C. T. Leondes. (New York, Academic Press).

Bull, H. O. (1957). Behavior: Conditioned Responses. Pp. 211-224 in The Physiology of Fishes, Vol. II, Ed. M. E. Brown (New York: Academic Press).

DALEN, J. (1973). Controlling Schools of Herring. Experiments 1973. 73-143-T, Div. Eng. Cybernetics, Norw. Inst. of Techn. (in Norwegian).

DiJkgraAF, N. (1962). The functioning and significance of the lateral-line organs. Biol. Rev., 38, 51-105.

Døving, K. B., Nordeng, H., and Enger, P. (1973). Electrophysiological studies on the olfactory sense in char (Salmo Alpinus). Comp. Biochem. Physiol., 45a, 21-24.

FesSARD, A. (1974). Handbook of Sensory Physiology, Vol. III/3. (New York, Springer Verlag).

Maniwa, Y., and HaShimoto, T. (1967). Research on the luring of fish shoals by utilizing underwater acoustical equipment. Proc. of the Second Symp. on Marine Bio-Acoustics, (New York: Pergamon Press).

MANTEYFel, B. P. (1970/1978). Biological bases for controlling fish behaviour. Transl. from Russian by National Marine Fisheries Service, Washington DC, U.S.A.

Mork, O. I., and BaLCHEN, J. G. (1980). Farming of salmon in fenced off open waters. STF48 F80043. Found. of Scient. and Ind. Research (SINTEF), Trondheim, Norway (in Norwegian).

Protasov, V. R. (1970). Vision and near orientation of fish. Israel Progr. for Scient. Translation, Jerusalem.

Radakov, D. V. (1973). Schooling in the ecology of fish (translated from Russian). John Wiley \& Sons.

Sternin, V. G., Nikonorov, I. V., and Bumeister, YU. K. (1976). Electrical fishing, theory and practice (translated from Russian), Israel Progr. for Scient. Translations, Jerusalem.

Sølvberg, E. (1977). An Electrical Barrier for Seawater. STF48 F77004. Found. of Scient. and Ind. Research (SINTEF), Trondheim, Norway (in Norwegian). 